\title{
Magnetic noise around metallic microstructures
}

\author{
Bo Zhang and C. Henkel \\ Institut für Physik, Universität Potsdam, 14469 Potsdam, Germany
}

(Dated: 28 Aug 2007)

\begin{abstract}
We compute the local spectrum of the magnetic field near a metallic microstructure at finite temperature. Our main focus is on deviations from a plane-layered geometry for which we review the main properties. Arbitrary geometries are handled with the help of numerical calculations based on surface integral equations. The magnetic noise shows a significant polarization anisotropy above flat wires with finite lateral width, in stark contrast to an infinitely wide wire. Within the limits of a two-dimensional setting, our results provide accurate estimates for loss and dephasing rates in so-called 'atom chip traps' based on metallic wires. A simple approximation based on the incoherent summation of local current elements gives qualitative agreement with the numerics, but fails to describe current correlations among neighboring objects.
\end{abstract}

PACS numbers: 44.40.+a Thermal radiation -05.40.-a Fluctuation phenomena, random processes, noise, and Brownian motion

\section{INTRODUCTION}

Thermal motion of charge carriers in a metallic object creates a randomly fluctuating magnetic field in the object's vicinity. These fields are relevant for many applications like high-precision measurements of biomagnetic signals $\stackrel{1}{ }$, nuclear magnetic resonance microscopy ${ }^{2}$, and miniaturized traps for ultra cold atoms $\mathrm{s}^{3.4}$. The original purpose of Purcell's influential 1946 paper $^{-5}$ was to point out that these magnetic fields have a spectral density that by far exceeds the Planck law for blackbody radiation at low frequencies. In fact, if only blackbody fields were present, magnetic dipole transitions between atomic or nuclear levels would never happen on laboratory time scales. The near fields sustained by material objects (that play the roles of sources and cavity) give the dominant contribution. Indeed, these near fields contain non-propagating (evanescent) components that are thermally excited as well and that dominate over free space radiation ${ }^{6.7}$. Phrased in another way, the dipole transition rate is enhanced because the elementary excitations in the metal provide additional decay channels 8.9 .

We focus in this paper on accurate calculations of magnetic field noise that are able to describe objects of arbitrary shape. Such objects occur, for example, in magnetic microtraps where complex networks of metallic wires create electromagnetic potentials with typical scales in the micron range ${ }^{3.4}$. The behaviour of the field spectrum, as the metallic geometry is changed, is far from intuitive. The spectral density increases with the material volume for small structures, but this trend saturates as soon as the typical scale gets larger than the penetration length (skin depth) of the fields in the material. It has even been found that a thin metallic layer can produce less noise than a half-space, depending on the ratios between observation distance, layer thickness, and skin depth, 70,11 . Experiments in the field of biomagnetism have shown significant changes when a metallic film is cut, at constant volume, into stripes ${ }^{1}$. Calculations for these cases necessarily require numerical methods to describe the propagation of magnetic fields both in vacuum and inside a metallic structure. This is the main topic of this paper. We also discuss previously developed approximations for planar structures and within the magnetostatic regime where analytical calculations are possi- ble. Our numerical methods are restricted here to two spatial dimensions (2D) where efficient solutions of Maxwell equations can be found with the help of boundary integral equations $12,13,14,15$. Our approach can also be combined with any other numerical method for field computations, permitting to cover three-dimensional cases as well.

The results we find can be summarized as follows. A planar structure (infinite lateral size) creates equal magnetic noise for all components of the magnetic field vector. This does not apply in three dimensions, but is specific to the two-dimensional setting we focus on here. Finite metallic objects show a strong anisotropy: the noise occurs preferentially along 'azimuthal' directions circling around the object. Increasing the amount of metallic material does not always give larger noise, in particular when the geometrical size becomes comparable to the skin depth. We find qualitative agreement with measurements of Ref 1 where thermal field fluctuations are reduced when a metallic object is split into disconnected pieces. The surface impedance approximation, that provides an accurate description of metallic reflectors for far-field radiation, is shown to be not reliable for observation distances shorter than the skin depth. Finally, qualitative (albeit not quantitative) agreement is obtained between our numerical data and an approximation based on the incoherent summation of fields generated by thermal current elements filling up the metallic volume. This method has been used in the interpretation of previous experiments 26,27 . Our results are, to our knowledge, the first quantitative test of this approximation in a nontrivial geometry.

The paper is organized as follows. We first review the link between the thermal radiation spectrum and classical dipole radiation (Sec I). Planar structures are analyzed in Sec III using the angular spectrum representation. We demonstrate in particular the isotropy of the magnetic noise spectrum and discuss the accuracy of the surface impedance approximation. $\mathrm{Sec}$ IV is devoted to our numerical scheme and to the results for single and multiple objects of rectangular shape. 


\section{MAGNETIC NEAR FIELD NOISE}

\section{A. Local noise power}

The fluctuations of the thermal magnetic field $\mathbf{B}(\mathbf{r}, t)$ are characterized by their local power spectral density (the Fourier transform of the autocorrelation function)

$$
\mathcal{B}_{i j}(\mathbf{r} ; \omega)=\int \mathrm{d} \tau \mathrm{e}^{\mathrm{i} \omega \tau}\left\langle B_{i}(\mathbf{r}, t) B_{j}(\mathbf{r}, t+\tau)\right\rangle .
$$

Higher moments are not needed for our purposes, and the average field (at frequency $\omega$ ) vanishes as is typical for thermal radiation. We assume the field to be statistically stationary so that the spectrum (1) does not depend on $t$. The diagonal tensor components $\mathcal{B}_{i i}(\mathbf{r} ; \omega)$ give the spectrum for a given cartesian component $B_{i}(\mathbf{r})$ or polarization direction. Previous work has shown a strong dependence on the position $\mathbf{r}$ near a metallic microstructure: power laws being the typical behaviour in the frequency range where the wavelength $\lambda=2 \pi c / \omega$ is much larger than the typical distances. For the temperature dependence, see Eq. (3) below. Our parameters of interest are: normal metallic conductors with temperatures above a few $\mathrm{K}, \mathbf{r}$ in the micron range and $\lambda$ of the order of centimeters or larger $(\omega / 2 \pi \leq 10 \mathrm{GHz})$. This upper limit on frequency corresponds to the strong magnetic dipole transitions in typical alkali atoms.) In this regime, the frequency dependence of the noise spectrum is weak and occurs via the material response (permittivity $\varepsilon(\omega)$ ). A characteristic length scale is the field penetration length (skin depth) $\delta$ defined by

$$
\frac{1}{\delta}=\frac{2 \pi}{\lambda} \operatorname{Im} \sqrt{\varepsilon(\omega)}=\sqrt{\frac{1}{2} \mu_{0} \omega \sigma(\omega)}
$$

where $\sigma(\omega)$ is the conductivity and $\mu_{0}$ the vacuum permeability. The second expression is based on the Hagen-Rubens approximation $\operatorname{Im} \varepsilon(\omega) \approx \sigma(\omega) /\left(\varepsilon_{0} \omega\right) \gg|\operatorname{Re} \varepsilon(\omega)|$. Within the Drude model for a metal, this is verified at frequencies much smaller than the charge carrier relaxation rate (in the $10^{15} \mathrm{~s}^{-1}$ range at room temperature). For highly conducting materials $(\mathrm{Au}, \mathrm{Ag}, \mathrm{Cu})$, this results in a skin depth of the order of $100 \mu \mathrm{m}(\omega / 2 \pi \mathrm{MHz})^{-1 / 2}$.

To compute the magnetic correlation spectrum, we use the fluctuation-dissipation theorem which is valid at thermal equilibrium (temperature $T)^{16}$

$$
\mathcal{B}_{i j}(\mathbf{r} ; \omega)=\frac{2 \hbar}{\mathrm{e}^{\hbar \omega / k_{B} T}-1} \operatorname{Im} \mathcal{G}_{i j}(\mathbf{r}, \mathbf{r} ; \omega)
$$

where $\mathcal{G}_{i j}\left(\mathbf{r}, \mathbf{r}^{\prime} ; \omega\right)$ is the Green function for the magnetic field, i.e., the field generated at $\mathbf{r}$ by a point magnetic dipole located at $\mathbf{r}^{\prime}$ and oscillating at the frequency $\omega, B_{i}(\mathbf{r}, t)=$ $\mathcal{G}_{i j}\left(\mathbf{r}, \mathbf{r}^{\prime} ; \omega\right) \mu_{j} \mathrm{e}^{-\mathrm{i} \omega t}+$ c.c.. This is actually a familiar result: the imaginary part of $\operatorname{tr} \mathcal{G}(\mathbf{r}, \mathbf{r} ; \omega)$ gives the local density of magnetic field modes, and the temperature dependent prefactor in Eq. (3) their average occupation number. The basic benefit of this formula is that it holds also for the full correlation tensor and even near material objects that absorb the field or generate thermal radiation. Generalizations to the nonequilibrium case exist (fields produced by a 'hot object' surrounded by a 'cold' environment) $)^{17,18}$, but are not needed for our purposes (see remarks in Sec $\mathrm{V}]$. We also note that the temperature dependent prefactor in Eq. (3) reduces to $2 k_{B} T / \omega$ for $T \geq 0.1 \mathrm{~K}$. In this limit, the order of field operators in the correlation function (1) becomes irrelevant. (The order we have adopted yields the rate of a magnetic dipole transition $i \rightarrow f$ with energy difference $E_{f}-E_{i}=\hbar \omega$.)

\section{B. Magnetic dipole radiation}

We are thus led to solve the following electrodynamic problem: find the complex magnetic field amplitude $\mathbf{B}(\mathbf{r} ; \omega \mid \boldsymbol{\mu})$ created by a monochromatic point dipole $\boldsymbol{\mu}(t)=\boldsymbol{\mu} \mathrm{e}^{-\mathrm{i} \omega t}+$ c.c. located at position $\mathbf{r}^{\prime}$. We then compute

$$
G_{i j}\left(\mathbf{r}, \mathbf{r}^{\prime} ; \omega\right)=\frac{\partial B_{i}(\mathbf{r} ; \omega \mid \boldsymbol{\mu})}{\partial \mu_{j}}
$$

In the limit $\mathbf{r} \rightarrow \mathbf{r}^{\prime}$ this field becomes the singular 'self field' and requires a cutoff in wavevector space. Its imaginary part is cutoff-independent, however, and given by $\operatorname{Im} \mathbf{B}\left(\mathbf{r}^{\prime} ; \omega\right)=$ $\mu_{0} \omega^{3} \boldsymbol{\mu} /\left(6 \pi c^{3}\right)$ (in three-dimensional free space).

The field $\mathbf{B}=\mathbf{B}(\mathbf{r} ; \omega \mid \boldsymbol{\mu})$ can be found from the vector potential A that solves the inhomogeneous Maxwell equation

$$
\nabla \times \nabla \times \mathbf{A}-k_{0}^{2} \varepsilon(\mathbf{r}) \mathbf{A}=\mu_{0} \nabla \times \boldsymbol{\mu} \delta\left(\mathbf{r}-\mathbf{r}^{\prime}\right),
$$

where $k_{0}=\omega / c$. The right-hand side is the current density corresponding to the magnetic dipole. There is no free charge density and we work in the gauge $\mathbf{E}=\mathrm{i} \omega \mathbf{A}$.

We now focus on the following geometry (fig 1 right): the position $\mathbf{r}^{\prime}$ of the source (i.e., where the magnetic noise spectrum is actually needed) is located in vacuum, and the metallic microstructures are filling a domain $\mathcal{D}$ where $\operatorname{Im} \varepsilon(\mathbf{r} ; \omega)=$ $\sigma(\mathbf{r} ; \omega) /\left(\varepsilon_{0} \omega\right)$ is nonzero (and large).
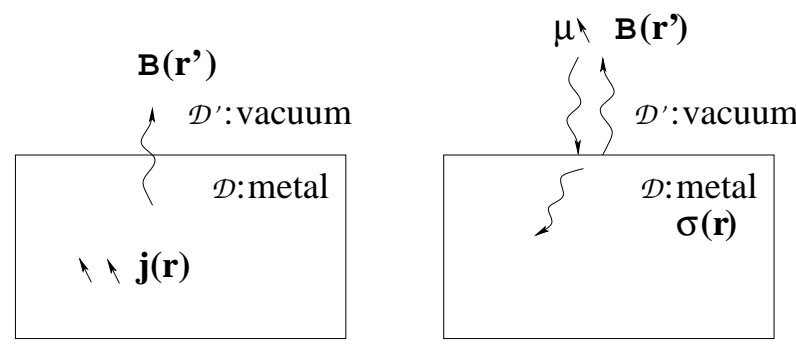

FIG. 1: Sketch of the considered geometry: (left) current fluctuations in a microstructure generate magnetic field fluctuations $\mathbf{B}\left(\mathbf{r}^{\prime}\right)$ at a position position $\mathbf{r}^{\prime}$ outside it. (right) The magnetic noise spectrum is calculated from the magnetic field radiated by a point magnetic dipole $\boldsymbol{\mu}$ located at $\mathbf{r}^{\prime}$. $\mathcal{D}$ and $\mathcal{D}^{\prime}$ : domains where the conductivity $\sigma(\mathbf{r} ; \omega)$ is nonzero or zero, respectively.

The outside domain is called $\mathcal{D}^{\prime}$. There, the vector potential satisfies an inhomogeneous Helmholtz equation with wavenumber $k_{0}$. All length scales we consider (distance dipole-microstructure $d$, object size) are much shorter than the wavelength so that $k_{0}$ is actually very small and can be neglected in a first approximation. This is the magnetostatic 
regime. (The finite value of $k_{0}$ is, of course, at the origin of the nonzero magnetic LDOS in free space.) We cannot make the magnetostatic approximation in $\mathcal{D}$ because there, we have a wavenumber $k_{0} \sqrt{\varepsilon(\mathbf{r} ; \omega)}=(1+\mathrm{i}) / \delta(\mathbf{r})$, and the (local) skin depth $\delta(\mathbf{r})$ is one of the characteristic length scales at hand. The fields in the domains $\mathcal{D}$ and $\mathcal{D}^{\prime}$ are connected by the usual matching conditions: the components of $\mathbf{A}$ tangential to the boundary are continuous, and $\mathbf{B}$ is continuous (the material is non-magnetic).

Eq.(5) provides a unique solution subject to the boundary condition that at infinity, the field behaves like an outgoing wave. In three [two] dimensions, this corresponds to a vector potential proportional to $\mathrm{e}^{\mathrm{i} k_{0} s} / s\left[\mathrm{e}^{\mathrm{i} k_{0} s} / \sqrt{s}\right]$ in the free space domain $\mathcal{D}^{\prime}$ when the distance $s=\left|\mathbf{r}-\mathbf{r}^{\prime}\right| \rightarrow \infty$ to the source becomes large compared to $\lambda$. In the magnetostatic limit $k_{0} \rightarrow 0$, the free space asymptotics is actually never reached at finite distances. The relevant boundary condition is then the same as for the scalar potential of an electric dipole: the vector potential goes to zero like $1 / s^{2}[$ like $1 / s]$ in three [two] dimensions, respectively.

Since we deal with a metallic object with $|\varepsilon| \gg 1$, it is tempting to perform the calculation based on the surface impedance boundary condition. The latter links the tangential components of magnetic field and vector potential by $B_{t}=-\mathrm{i} \omega Z A_{z}$, where $\omega Z=(1+\mathrm{i}) / \delta$. Note that this is a local relation that can only hold if the scale of variation of the fields on the object surface is much larger than the skin depth $\delta$. In the present study, a point-like source illuminates the object with its near field $\left[A_{\text {bulk }}\left(\mathbf{r}-\mathbf{r}^{\prime}\right)\right.$ in Eq.(7)], and this field shows a typical extension of the order of the objectsource distance $d$. The surface impedance approximation is hence expected to break down for $d \ll \delta$. We shall confirm this explicitly for the planar structures discussed in the following Sec III

\section{RESULTS: LAYER}

\section{A. Two dimensions}

In this paper, we focus on a two-dimensional (2D) geometry to simplify the numerical calculations described in SecIV The magnetic moment is chosen in the computational plane (the $x y$-plane), as shown in Fig 1. Adapting the wave equation (5) to two dimensions, we find that the vector potential has a single nonzero component that points out of the plane. We then work with a scalar function $A(\mathbf{r})=A(x, y)$ that solves

$$
\nabla^{2} A+k_{0}^{2} \varepsilon(\mathbf{r}) A=\mu_{0}\left(\mu_{y} \partial_{x^{\prime}}-\mu_{x} \partial_{y^{\prime}}\right) \delta\left(\mathbf{r}-\mathbf{r}^{\prime}\right)
$$

In a homogeneous medium ('bulk'), the solution with the appropriate boundary conditions is

$$
A_{\text {bulk }}\left(\mathbf{r}-\mathbf{r}^{\prime}\right)=\frac{i \mu_{0}}{4}\left(\mu_{y} \partial_{x^{\prime}}-\mu_{x} \partial_{y^{\prime}}\right) H_{0}\left(k_{0} \sqrt{\varepsilon}\left|\mathbf{r}-\mathbf{r}^{\prime}\right|\right)
$$

where $H_{0}$ is the Bessel function of the third kind (Hankel function), usually denoted $H_{0}^{(1)}=J_{0}+\mathrm{i} Y_{0}$. ¿¿ $i$ From this, we get the magnetic field by taking the 'curl', $B_{x}=\partial_{y} A$, $B_{y}=-\partial_{x} A$. The resulting self field in free space is

$$
\operatorname{Im} \mathbf{B}\left(\mathbf{r}^{\prime} \mid \boldsymbol{\mu}\right)=\frac{1}{8} \mu_{0} k_{0}^{2} \boldsymbol{\mu}
$$

provided the dipole $\boldsymbol{\mu}$ is real. In the magnetostatic limit, this field is negligibly small. The bulk solution (7) then goes over into

$$
A_{\text {bulk }}\left(\mathbf{r}-\mathbf{r}^{\prime}\right) \approx-\frac{\mu_{0}}{2 \pi} \frac{\left(x-x^{\prime}\right) \mu_{y}-\left(y-y^{\prime}\right) \mu_{x}}{\left|\mathbf{r}-\mathbf{r}^{\prime}\right|^{2}}
$$

This equation describes the field with which the dipole 'illuminates' the sample. Note that it is scale-free: the typical 'spot size' on the microstructure is only determined by the distance $d$ between dipole and top surface.

\section{B. Reflected field}

In this section, we consider that the boundary of the medium is the plane $y=0$; the field at the source point $\mathbf{r}^{\prime}=(0, d)$ is then related to the (Fresnel) reflection coefficients from the surface. We expand the solution to Eq.(6) in plane waves (wavevector $k$ parallel to the boundary) and have above the medium $(y>0)$ :

$$
\begin{aligned}
A(x, y)= & \mu_{0}\left(\mu_{x} \partial_{y^{\prime}}-\mu_{y} \partial_{x^{\prime}}\right) \int_{-\infty}^{+\infty} \frac{\mathrm{d} k}{2 \pi} \frac{\mathrm{e}^{\mathrm{i} k\left(x-x^{\prime}\right)}}{2 \kappa} \\
& \times\left(\mathrm{e}^{-\kappa\left|y-y^{\prime}\right|}+r(k) \mathrm{e}^{-\kappa\left(y+y^{\prime}\right)}\right)
\end{aligned}
$$

where $\kappa=\sqrt{k^{2}-k_{0}^{2}}$. (The square root is chosen such that $\operatorname{Re} \kappa \geq 0$.) The coefficient $r(k)$ describes the reflection of the field from the medium boundary. It is given by the Fresnel formula

$$
r(k)=r_{\text {half space }}(k) \equiv \frac{\kappa-\kappa_{\mathrm{m}}}{\kappa+\kappa_{\mathrm{m}}}, \quad \kappa_{\mathrm{m}}=\sqrt{k^{2}-2 \mathrm{i} / \delta^{2}}
$$

for a medium with skin depth $\delta$ filling the half-space $y<0$. For a layer (thickness $h$ ) on top of a substrate, we have

$$
r_{\text {layer }}(k)=\frac{r_{\text {top }}+r_{\text {bottom }} \mathrm{e}^{-2 \kappa_{\mathrm{m}} h}}{1-r_{\text {top }} r_{\text {bottom }} \mathrm{e}^{-2 \kappa_{\mathrm{m}} h}}
$$

where $r_{\text {top }}=r_{\text {half space }}$ is given by Eq.(11) and $r_{\text {bottom }}$ describes the reflection from the layer-substrate interface. It is given by Eq.(11) with the replacements $\kappa \mapsto \kappa_{\mathrm{m}}, \kappa_{\mathrm{m}} \mapsto \kappa_{\mathrm{s}}=$ $\left(k^{2}-\varepsilon_{\mathrm{s}} k_{0}^{2}\right)^{1 / 2}$ where $\varepsilon_{\mathrm{s}}$ is the substrate permittivity.

All the relevant information for the magnetic noise power is contained in the reflection coefficient $r(k)$. In fact, when the integral in (10) is performed and the imaginary part taken, it turns out that the reflected waves (second term) dominate over the free space contribution (first term) by at least a factor $\lambda^{2} \delta / d^{3} \gg 1$. This is connected to the fact that the relevant wavenumbers $k$ for our problem are of the order of $1 /\left(y+y^{\prime}\right)=1 /(2 d)$ which is much larger than $k_{0}$. We can 
hence apply the approximation $\kappa \approx|k|$. The reflection coefficient (11) for the metallic half-space then depends only on the parameter $k \delta$. For the metallic layer geometry, we focus for simplicity on a substrate whose conductivity is much smaller than in the metal. The influence of the substrate has been studied in Ref 19: already a ratio of 10 to 100 between the substrate and layer conductivities is sufficient to make the substrate behave like vacuum. We then have $r_{\text {bottom }} \approx-r_{\text {top }}$ in Eq. 12.

\section{Polarization dependence}

Let us analyze first the dependence on the orientation of the source dipole. If $\boldsymbol{\mu}$ is perpendicular to the medium (only $\left.\mu_{y} \neq 0\right)$, the reflected field is given by

$$
B_{y}\left(\mathbf{r} \mid \mu_{y}\right)=\mu_{0} \mu_{y} \int_{-\infty}^{\infty} \frac{\mathrm{d} k}{2 \pi} \frac{k^{2}}{2 \kappa} r(k) \mathrm{e}^{\mathrm{i} k\left(x-x^{\prime}\right)} \mathrm{e}^{-\kappa\left(y+y^{\prime}\right)}
$$

The limit $\mathbf{r} \rightarrow \mathbf{r}^{\prime}$ yields an imaginary part

$$
\operatorname{Im} B_{y}\left(\mathbf{r}^{\prime} \mid \mu_{y}\right)=\mu_{0} \mu_{y} \operatorname{Im} \int \frac{\mathrm{d} k}{2 \pi} \frac{k^{2}}{2 \kappa} r(k) \mathrm{e}^{-2 \kappa d} .
$$

Repeating the calculation for a parallel dipole, we find for $\operatorname{Im} B_{x}\left(\mathbf{r}^{\prime} \mid \mu_{x}\right)$ the same expression as Eq. (14), and consequently the noise spectrum is isotropic, $\mathcal{B}_{x x}=\mathcal{B}_{y y}$. This is a remarkable property of a laterally infinite structure in $2 \mathrm{D}$. (In $3 \mathrm{D}$, the polarization perpendicular to a planar interface has a noise power twice as large as the parallel polarization ${ }^{7,20}$.) We show below that a significant polarization anisotropy arises above a metallic wire of finite width.

\section{Wavevector dependence}

The reflection coefficient (12) is plotted in Fig 2 for typical layers. Consider first a thickness larger than a few skin depths. One observes a maximum value of its imaginary part (relevant for the magnetic LDOS) when the decay constant $\kappa \approx k$ is matched to $1 / \delta$. This is confirmed by an asymptotic analysis whose results are given in Table I. (See Refs 201 for details on the asymptotic expansion.) One of the two limiting cases (namely $k_{0} \ll k \ll 1 / \delta$ ) corresponds precisely to the surface impedance approximation where the reflection coefficient 11) is approximated by

$$
r(k) \approx-1+(1+\mathrm{i})|k| \delta
$$

Here, the skin depth is much smaller than the lateral period and the field barely penetrates into the material. Fig 2 and Table @ show strong deviations in the opposite regime $k \gg$ $1 / \delta$ that is relevant at distances $d \ll \delta$.

Consider now a layer much thinner than the skin depth. ¿FFrom Fig,2, different regimes can be read off that are separated on the $k$-axis by the scales $h / \delta^{2} \ll 1 / h$, as can be seen in Fig It is worth noting that for small $k$, thin layers

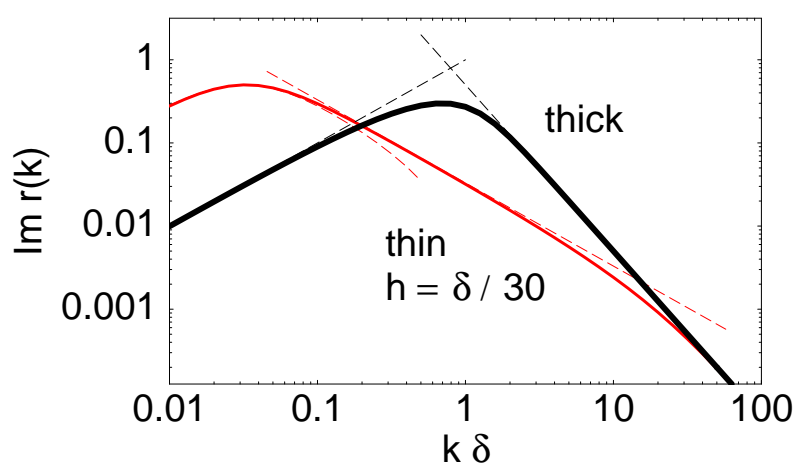

FIG. 2: Reflection coefficients 11, 12 for thin and thick metallic layers. We plot the imaginary part only. The dashed lines represent the formulas of Table $\prod$ The wavenumber is scaled to the inverse skin depth $1 / \delta$. For the thick layer, $h=\infty$. We take the conductivity of gold at room temperature and a frequency $\omega / 2 \pi \approx 1.1 \mathrm{MHz}$ in all figures. This leads to the value $\delta=71 \mu \mathrm{m}$ and a vacuum wavelength $\lambda / \delta \approx 3.8 \times 10^{6}$.

\begin{tabular}{|c|c|c|c|}
\hline & $k \ll 1 / \delta$ & $1 / \delta \ll k$ & \\
\hline $\operatorname{Im} r_{\text {half space }}(k)$ & $k \delta$ & $\frac{1}{2 k^{2} \delta^{2}}$ & \\
\hline \hline & $k \ll 1 / \delta$ & $h / \delta^{2} \ll k \ll 1 / h$ & $1 / h \ll k$ \\
\hline $\operatorname{Im} r_{\text {layer }}(k)$ & $\operatorname{Im} \frac{-1+(1+\mathrm{i}) k \delta}{1+\mathrm{i} k \delta^{2} / h}$ & $\frac{h}{k \delta^{2}}$ & $\frac{1}{2 k^{2} \delta^{2}}$ \\
\hline
\end{tabular}

TABLE I: Asymptotic approximations to the reflection coefficients from a half-space and a layer. We distinguish between thin (thickness $h \ll \delta)$ and thick layers ( $h \geq \delta$, 'half space'). The first and second columns (thin layer) overlap in an intermediate $k$-range (see Fig 2). The magnetostatic limit $k_{0} \ll k$ is taken throughout. These formulas are plotted as dashed lines in Fig 2 .

show even larger losses [ $\operatorname{Im} r(k)]$ than thick ones; the maximum is shifted towards the smaller value $k \sim h / \delta^{2}$ and has a larger amplitude. This behaviour has been recognized before in magnetic noise studies in the $\mathrm{kHz}$ range ${ }^{7}$. In the infrared range, it is also well known that the absorption by a metallic layer can be optimized at a specific thickness. (See, e.g., $\operatorname{Ref} 22$ for incident far-field radiation where $|k| \leq k_{0}$.) Conversely, for a given thickness $h$ and dipole distance $d$, the magnetic noise power shows a maximum as the skin depth is changed $^{7,10}$. This 'worst case' occurs when the characteristic wavevector $1 / d$ is matched to $h / \delta^{2}$.

\section{E. Distance dependence}

The asymptotics in $k$-space translate into power laws for the dependence of the magnetic power spectrum $\mathcal{B}_{i i}(d ; \omega)$ on distance $d$, as shown in Fig 3 In fact, the integrand in Eq.(14) peaks around $k \sim 1 /(2 d)$, and the result of the integration is determined, to leading order, by the behaviour of $r(k)$ in this range. We thus find the power laws summarized in Table $\amalg$ and visible in Fig 3 We use as convenient 


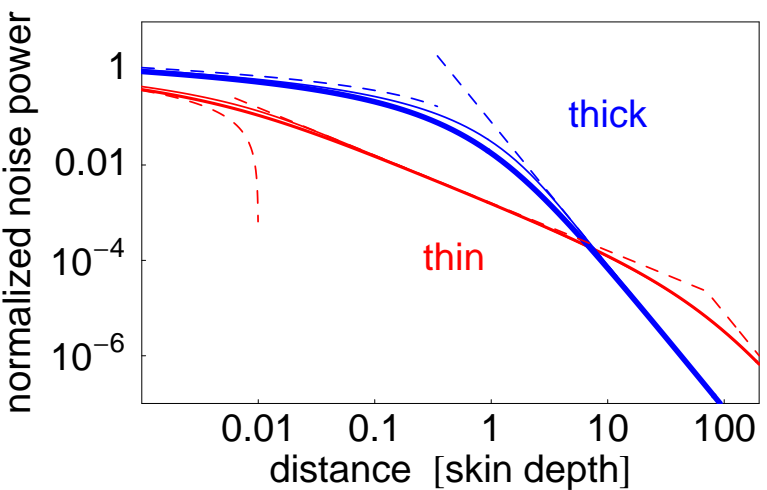

FIG. 3: Local magnetic noise power $\mathcal{B}_{i i}(d ; \omega)$ vs. distance from metallic layer in double logarithmic scale (2D calculation). Top curve [blue]: thick layer; bottom curve [red]: thin layer. The dashed lines give the leading order power laws of Table $\amalg$ The thick curves arise from the numerical integration of Eq. 10, the thin curves are an interpolation formula described in the text.

The magnetic noise power is isotropic above a planar structure in $2 \mathrm{D}$ (the perpendicular and parallel field components have the same power). It is scaled to $\mu_{0} k_{B} T /\left(\omega \delta^{2}\right)$, and the distance is scaled to the skin depth $\delta$. Thin [thick] layer: $h=0.01 \delta[3 \delta]$.

\begin{tabular}{|c|c|c|c|}
\hline & $d \ll \delta$ & $\delta \ll d$ & \\
\hline $\mathcal{B}_{i i \text {,half space }}(d)$ & $\frac{\log (\delta / d)}{2 \pi}$ & $\frac{\delta^{3}}{4 \pi d^{3}}$ & \\
\hline \hline & $d \ll h \ll \delta$ & $h \ll d \ll \delta^{2} / h$ & $\delta^{2} / h \ll d$ \\
\hline $\mathcal{B}_{\text {ii,layer }}(d)$ & $\frac{\log (h / d)}{2 \pi}$ & $\frac{h}{2 \pi d}$ & $\frac{\delta^{4}}{4 \pi h d^{3}}$ \\
\hline
\end{tabular}

TABLE II: Power laws for the magnetic noise spectrum in two dimensions above a half space and a thin metallic layer (dashed lines in Fig 3). The noise spectrum is given in units of $\mu_{0} k_{B} T /\left(\omega \delta^{2}\right)$.

unit in all the plots the noise level $\mu_{0} k_{\mathrm{B}} T /\left(\omega \delta^{2}\right)$. Normalized to blackbody radiation (in $2 \mathrm{D}$ free space), this level is $\left(2 /\left(k_{0} \delta\right)\right)^{2} \sim 1.5 \times 10^{12}$ at $1.1 \mathrm{MHz}$ for gold at room temperature, a striking illustration of the Purcell effect ${ }^{5}$. A common trend is that the magnetic noise power increases as the metallic medium is approached. As the distance $d$ is getting much smaller than the thickness $h$, thin and thick layers behave the same, as expected. At larger distances, but still smaller than the skin depth, the noise power is proportional to the volume of metallic material, hence to the layer thickness ${ }^{3,23}$. This trend is reversed for $d>\delta \sqrt{\delta / 2 h}$ where thin layers give a larger noise level than thick ones, 7,10 .

A reasonably accurate approximation that interpolates between these power laws can be found by performing the $k$ integral using the asymptotic formulas of Table $\Pi$ in their respective domains of validity. The result is a sum of incomplete gamma functions $\Gamma\left(n, x, x^{\prime}\right)(n=0,1,2)$ whose arguments are, for example, $x \sim d h / \delta^{2}, x^{\prime} \sim d / \delta$, or $d / h$ (thin lines in Fig 3 and Appendix A. We have checked that the asymptotics of the gamma function reproduce the power laws summarized in Table [I] There are regimes where the sub-leading terms give significant corrections, in particular in the transition re- gions between the power laws.

Finally, the surface impedance approximation gives a magnetic noise that is represented in Fig 3 by the dashed line close to the 'thick layer' for $d>\delta$. The agreement with the full calculation in this range is expected: the 'illuminating field' is getting more and more uniform on the scale of the skin depth. At shorter distances, the surface impedance approximation severely overestimates the noise level because it cannot describe properly the field variations on scales smaller than $\delta$. For the thin layer, the conventional surface impedance approach gives a wrong result even if $d>\delta$ because top and bottom surfaces do not decouple from each other. This can be repaired using effective (thickness-dependent) surface impedances, see, e.g., Ref 24 and citations therein.

\section{RESULTS: FINITE SIZE OBJECTS}

We now describe numerical calculations that we have performed to assess the importance of the finite lateral size of the metallic structure. This is particularly relevant, for example, in atom chips where a continuous metallic layer is etched to define wires that can be addressed with different currents $s^{3.4,25}$. It is actually desirable to minimize the amount of metallic material, leaving just a few wires to create the fields for atom trapping. In fact, it has been argued that the magnetic noise power roughly scales with the metallic volume as long as the characteristic distances are smaller than the skin depth ${ }^{3,23}$. For laterally finite structures, this claim as well as other calculations have been based so far on approximate methods that fail to reproduce even the planar layer to within a factor of two or three $11,23,26,27$. The numerical results we describe here are a first step towards an accurate estimate of magnetic noise power near structures of finite size.

\section{A. Boundary integral equations}

Within the assumption of near field radiation being in equilibrium with the metallic object, we compute the noise power from the magnetic Green function in Eq.(3). The magnetic field radiated by a point source and reflected by the object solves the wave equation (6). We reformulate the wave equation in terms of boundary integral equations. This has been described elsewhere ${ }^{12,13,14,15}$, and we quote only the basic formulas here. Our unknowns are the nonzero component $A$ of the vector potential and its normal derivative $F=\partial A / \partial n \equiv$ $\mathbf{n} \cdot \nabla A$ on the object surface $\mathcal{S}$, where $\mathbf{n}$ is the outward unit normal to $\mathcal{S}$. Both quantities are continuous (actually, $F$ is equal to the tangential magnetic field) and can be found from the system of integral equations

$$
\begin{aligned}
& A(\mathbf{r})=2 A_{\text {bulk }}\left(\mathbf{r}-\mathbf{r}^{\prime}\right)- \\
& 2 \oint \mathrm{d} a(\mathbf{x})\left[G_{1}(\mathbf{r}-\mathbf{x}) F(\mathbf{x})-\frac{\partial G_{1}}{\partial n}(\mathbf{r}-\mathbf{x}) A(\mathbf{x})\right](16) \\
& A(\mathbf{r})= \\
& 2 \oint \mathrm{d} a(\mathbf{x})\left[G_{\varepsilon}(\mathbf{r}-\mathbf{x}) F(\mathbf{x})-\frac{\partial G_{\varepsilon}}{\partial n}(\mathbf{r}-\mathbf{x}) A(\mathbf{x})\right](17)
\end{aligned}
$$


where $A_{\text {bulk }}\left(\mathbf{r}-\mathbf{r}^{\prime}\right)$ is given in Eq.(7). Both the observation point $\mathbf{r}$ and the integration points $\mathbf{x}$ are taken on the object boundary $\mathcal{S}$ here, $\mathrm{d} a(\mathbf{x}))$ being the surface element. We use the scalar Green functions [see Eq.(7)]

$$
G_{\varepsilon}(\mathbf{r})=\frac{\mathrm{i}}{4} H_{0}\left(k_{0} \sqrt{\varepsilon}|\mathbf{r}|\right) \text {. }
$$

If we would take the magnetostatic limit, $G_{1}(\mathbf{r}) \rightarrow$ $-(2 \pi)^{-1} \log |\mathbf{r}|$, the Green functions in vacuum and in the medium would differ (in sub-leading order) by a constant, leading to inconsistencies. We avoid this by retaining the finite value of $k_{0}$ even for the vacuum Green function. The integrals (16, 17) are to be understood as principal values to handle the singularities of the Green functions as $\mathbf{x} \rightarrow \mathbf{r}$. We discretize them on a finite element decomposition of the object boundary, as described in Ref 12 . The resulting linear system is solved with standard numerical tools. Once the fields $A$, $F$ are known on the surface, the reflected field at the source position $\left(\mathbf{r}^{\prime}\right)$ can be found from

$$
\begin{aligned}
& A_{\mathrm{ref}}\left(\mathbf{r}^{\prime}\right)= \\
& -\oint \mathrm{d} a(\mathbf{x})\left[G_{1}\left(\mathbf{r}^{\prime}-\mathbf{x}\right) F(\mathbf{x})-\frac{\partial G_{1}}{\partial n}\left(\mathbf{r}^{\prime}-\mathbf{x}\right) A(\mathbf{x})\right]
\end{aligned}
$$

Note that $G_{1}$ and $\partial G_{1} / \partial n$ are both essentially real here (the imaginary parts scale with $k_{0}$ ). The magnetic noise, via $\operatorname{Im} \mathbf{B}\left(\mathbf{r}^{\prime}\right)$, is thus determined by the imaginary parts of $A$ and $F$ on the object boundary. This is not surprising since the induced current density is $\sigma E=\mathrm{i} \omega \sigma A$.

\section{B. Single wire}

We have solved the integral equations for rectangular wires of thickness $h$ and width $w$. In a first step, we have validated our numerical scheme by comparing flat, wide wires $(w \gg h)$ to the infinite layer results of Sec III Typical plots are shown in Fig, 4 where the magnetic noise power (symbols) is plotted vs. the distance $d$ above the wire centre. Good agreement with the analytical results for an infinitely wide wire (solid lines) is only obtained at short distance, where for geometrical reasons the wire appears wider. At distances above $20 \mu \mathrm{m}$, the deviations start to grow. In all the plots, we take a skin depth $\delta=70 \mu \mathrm{m}$. The slow convergence in the limit $w \rightarrow \infty$ can be attributed to the long-range behaviour of the fields; this is more pronounced in two dimensions compared to three. Note in particular the strong splitting between the two polarization directions for the thick wire that does not occur above an infinitely wide wire in 2D (Sec IIIC). Interestingly, the $y$-component shows more noise above a thick wire while this tendency is reversed above a thin wire. This polarization anisotropy could provide a tool to improve the lifetime in a magnetic trap: one orients the static trapping field parallel to direction of the strongest noise. (In fact, trap loss and spin flips are induced by magnetic fields perpendicular to the static trap field.) The choice of a trapping field along the weak noise direction is favorable if one wants to reduce the dephasing rate of the trapped spin states (generated by fluctuations of the Larmor frequency, see Ref $\frac{3}{2}$ ).

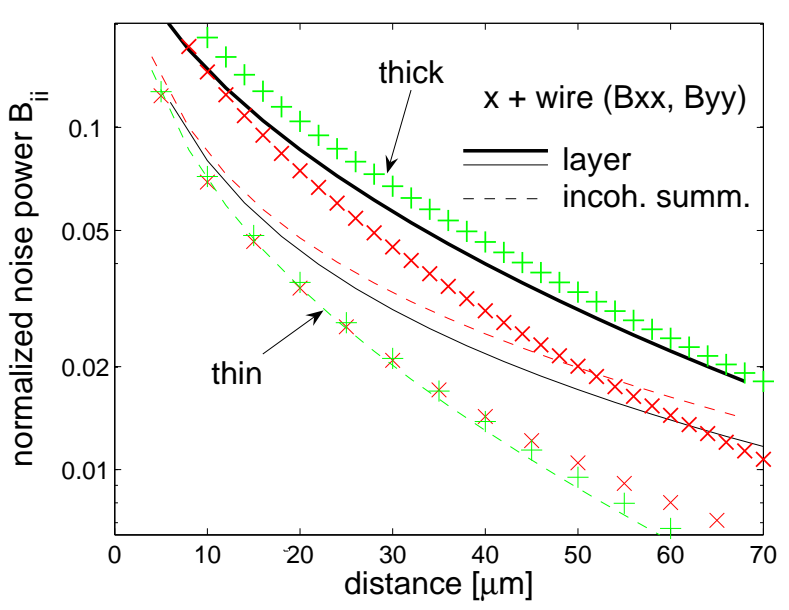

FIG. 4: Magnetic noise spectra $\mathcal{B}_{i i}(d)$ vs. distance $d$ above the centre of thin and thick metallic wires. Symbols $+(\times)$ : numerical calculation for the $\mathcal{B}_{y y}\left(\mathcal{B}_{x x}\right)$ component (see Fig 1 ). Solid lines: infinitely wide wire (layer), as computed in Sec III Dashed lines: incoherent summation (thin layer only, upper curve: $\mathcal{B}_{x x}$ ), see Sec. IVC Thin wire: width and thickness $200 \times 7 \mu \mathrm{m}$; thick wire: $200 \times 160 \mu \mathrm{m}$. The skin depth is $\delta=70 \mu \mathrm{m}$.

Another finite-size effect is shown in Fig $[5]$ where the position is varied parallel to the top surface of a thin wire. Above the centre of wide wires, the noise levels are nearly constant (not shown). Beyond the wire edges, one observes a sharp drop in $\mathcal{B}_{x x}$, with a characteristic scale fixed by the distance. The $y$-component shows a broad maximum near the edge that is more pronounced for narrow wires. This is due to a gradually changing direction of maximum noise that is 'azimuthal' with respect to the object, as expected for magnetic fields generated by currents flowing perpendicular to the computational plane (see inset of Fig 5). We find the direction of maximum noise by looking for the eigenvectors of the $2 \times 2$ matrix $\mathcal{B}_{i j}$. This matrix can be shown to be symmetric (reciprocity), and the eigenvectors that are not orthogonal very near to the wire's corner, are an artefact of our numerical method that converges very slowly at these points.

In Figs 6,7 the thickness of the wire is changed with the observation point remaining above the centre. We observe an approximately linear increase with the width that saturates slowly. We also note that $\mathcal{B}_{x x}$ (left) levels off faster than $\mathcal{B}_{y y}$ (right). The difference between Fig 6 and Fig 7 is the distance of observation: at short distance (Fig 6), the largest widths show a noise power fairly close to the planar layer limit (cf. the symbols at the right end). At distances comparable to the skin depth (Fig.7), the deviations from the planar layer limit (symbols) are still large. Note also that the noise has dropped in amplitude and that the increase with width is slower.

\section{Incoherent summation}

This behaviour can be qualitatively understood using the 'incoherent summation' approximation developed in Ref 23: 

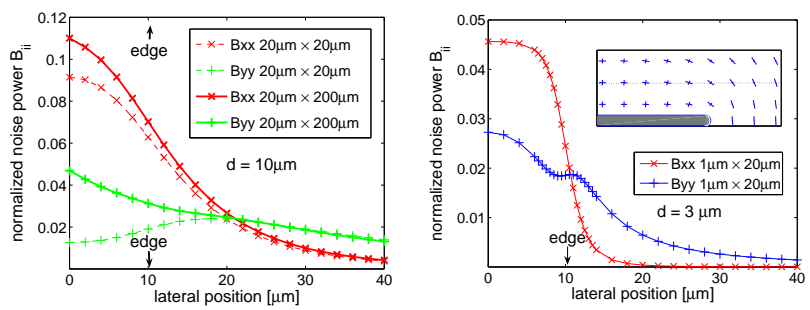

FIG. 5: Magnetic noise spectra $\mathcal{B}_{i i}(d)$ vs. lateral position, at a fixed distance $d$. The arrows mark the edges of the wires. Symbols $\times[+]$ : spectrum $\mathcal{B}_{x x}\left[\mathcal{B}_{y y}\right]$ parallel [perpendicular] to the top face of the wire. Skin depth: $\delta=70 \mu \mathrm{m}$.

Left panel: thickness and width are $20 \times 200 \mu \mathrm{m}$ (wide wire) and $20 \times 20 \mu \mathrm{m}$ (narrow wire). Distance $d=10 \mu \mathrm{m}$. Right panel: thickness and width are $1 \times 20 \mu \mathrm{m}$, distance $d=3 \mu \mathrm{m}$ (see dotted line of inset). Inset: illustration of anisotropic noise near the wire edge. The crosses are oriented along the polarization vectors that show maximum and minimum noise, the 'arm lengths' being proportional to the rms noise. The magnetic field noise is dominantly azimuthal, with field lines circling around the wire. The dotted line $(d=3 \mu \mathrm{m})$ shows the positions scanned through in the right panel.
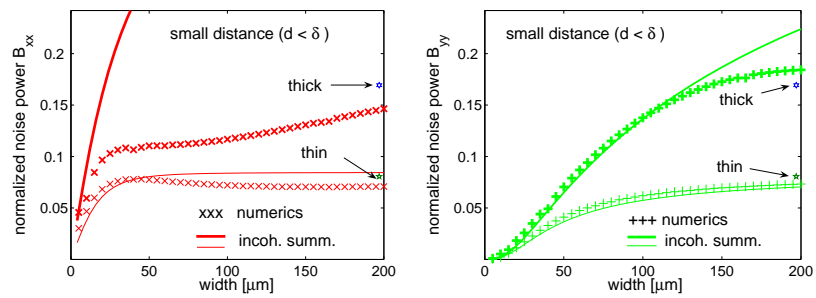

FIG. 6: Magnetic noise spectra vs. the width of a rectangular wire. (left) $x$-polarization, parallel to the top face; (right) $y$-polarization. Symbols: numerical calculations; solid lines: incoherent summation approximation (see Sec IV C). The symbols on the right margin give the values for an infinitely wide wire (layer). The observation point is located above the wire centre, at a distance $d=10 \mu \mathrm{m}$. The wire thickness is $7 \mu \mathrm{m}$ (thin) and $160 \mu \mathrm{m}$ (thick). Skin depth: $\delta=$ $70 \mu \mathrm{m}$.

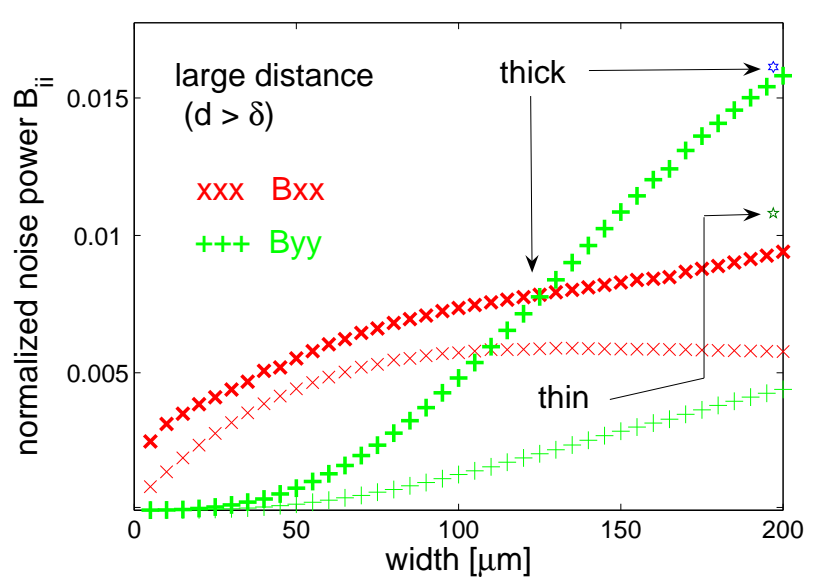

FIG. 7: Same as Fig 6 but at an observation distance $d=75 \mu \mathrm{m}$. Results from the incoherent summation are not shown, as they strongly deviate.
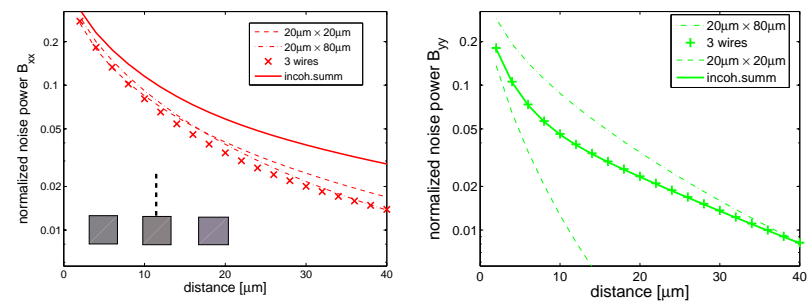

FIG. 8: Noise power generated by three wires, as a function of distance (see inserted sketch, with the dashed line illustrating the observation points). The wires have a quadratic cross section $20 \mu \mathrm{m} \times$ $20 \mu \mathrm{m}$ and are separated by a gap of $20 \mu \mathrm{m}$. The noise is measured above the center of the central wire. Left panel: horizontal polarization, right panel: vertical polarization. Symbols: numerical result; solid line: incoherent summation. For comparison is shown: a single wire of same cross section (dashed line) and a wide wire $20 \mu \mathrm{m} \times 80 \mu \mathrm{m}$ with approximately the same volume (dash-dotted line). Skin depth $\delta=70 \mu \mathrm{m}$.

the metallic volume is broken into mutually incoherent point current elements whose magnetic fields are computed within magnetostatics and neglecting the presence of the metallic object. We give the resulting formulas for two dimensions in Appendix $B$, the integrals are solved by special functions for a wire with rectangular cross section. The solid lines in Fig 6 demonstrate that incoherent summation gives a reliable approximation if the skin depth is the largest length scale (not true for the thick wire). The noise power always increases with the metallic volume within this approximation, however, and it may also happen that a wider wire produces a slightly weaker noise ( $x$-polarized curve for a thin wire in Fig 6). This is qualitatively similar to the trend of Fig 3 where a thick layer can produce less noise than a thin one at distances larger than the skin depth. The polarization anisotropy is also qualitatively reproduced by the incoherent summation method, although $\mathcal{B}_{x x}$ is overestimated. In fact, due to damping on the scale of $\delta$, not the entire volume of the thick layer contributes to the noise. The dashed lines in Fig 4 and further calculations show that the quantitative agreement is systematically better for the field component perpendicular to the nearest metal surface (here, $\mathcal{B}_{y y}$ ).

\section{Multiple wires}

This is the generic situation in miniaturized magnetic traps ('atom chips') with wires being defined by etchings in a metallic layer. We consider three wires of identical cross section and smaller than the skin depth. We show in Fig 8 the dependence on the vertical distance, above the central wire. Our results interpolate smoothly between a single narrow wire $(d \ll w)$ and a single wide wire $(d \gtrsim 2 w)$, as could have been expected. In fact, the three geometries give nearly the same noise in the azimuthal $\left(B_{x}\right)$ polarization. The incoherent summation approximation overestimates this noise component (similar to Fig 6). We attribute this to correlations between the current fluctuations in neighboring wires that are not 

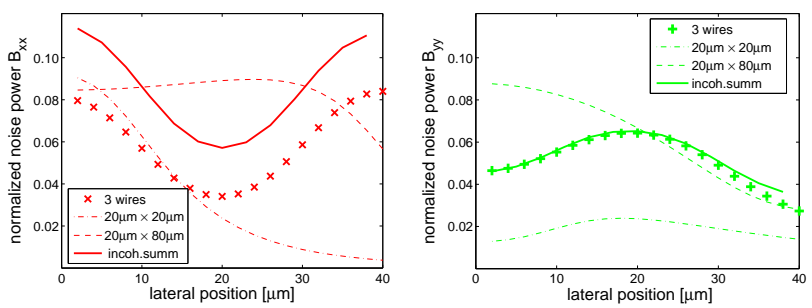

FIG. 9: Same as Fig 8 , but at fixed distance $d=10 \mu \mathrm{m}$, scanning the lateral position. $x=0$ is above the center of the central wire).

captured by incoherent summation. On the other hand, this approximation gives an excellent agreement for the weaker noise component $B_{y}$.

When we shift the observation point laterally, along the axis connecting the wire centres, we get Fig 9 . The stronger $B_{x^{-}}$ polarization shows maxima of noise above each wire, as expected. In the $B_{y}$-polarization, a maximum occurs in the gap between the wires. This conforms to the general trend of 'azimuthal noise' illustrated in Fig 5 (inset). It is also interesting that above the central wire $(x=0)$, three wires generate less noise than only one and also less than a single wide wire (approximately a merger of the three). This observation goes into the same direction as the experiments reported by Nenonen and co-workers 1 where a reduction of thermal magnetic fields was achieved by cutting a metallic film into stripes. We attribute this behaviour to negative correlations between the currents in neighboring wires brought about by the propagation of the magnetic field between them. In fact, the noise could only increase if the wire currents were strictly uncorrelated.

The performance of the incoherent summation approximation (solid lines) can be clearly seen, the trends being similar to Fig, good agreement for the $B_{y}$-polarization, overestimation of the perpendicular case due to the neglect of correlation effects.

\section{CONCLUSIONS}

We have described in this paper numerical and analytical results for the thermal fields surrounding a two-dimensional metallic object of arbitrary cross-section. The role of the skin depth $\delta$ as a characteristic length scale has been highlighted. At distances smaller than $\delta$, the spectral noise power roughly scales with the volume of the metallic material (Figs. 3, 6, 7). We have reviewed a simple method based on this idea, the 'incoherent summation approximation'. It systematically overestimates the noise power in one of the two field polarizations, but otherwise reproduces the main features as long as the skin depth is the largest scale. The strong polarization anisotropy that we have found suggests strategies to minimize loss or decoherence due to thermal magnetic fields, as observed in recent experiments $19,26,28$ : this can be done by suitably choosing the direction of the static trapping fields. We have also shown that the noise power can be significantly non-additive when dealing with multiple objects. This could be relevant for the discrepancy between experiment and theory observed in Ref 19, although our method (restricted to 2D) do not permit quantitative predictions of trap lifetimes.

We now comment on possible extensions of this analysis. Our framework is also able to provide an approximate description of superconducting structures. In fact, since we deal with magnetic field fluctuations at a finite frequency, there is always some penetration into the superconductor or, equivalently, a finite resistivity. This can be attributed to a fraction of carriers in a normally conducting state. Calculations for superconductors of planar geometry have been reported in Refs 10,29, with applications for miniaturized atom traps in mind. More accurate descriptions require one to solve the London equations at finite frequency inside the superconductor, using for example the two-fluid mode ${ }^{30}$.

We recall that we use in this paper a local version of Ohm's law. For very pure metallic films, the ballistic transport of charge carriers implies a nonlocal response $31,32,33,34$. This may be particularly relevant for wires defined by doping a semiconductor, but would require major changes for the numerical approach of SecIV

Finally, a brief remark on non-equilibrium settings. Consider an isolated metallic object held at a temperature different from its surroundings (materialized by the vacuum chamber walls, for example). By applying the generalized Kirchhoff relations (see, e.g., Ref 17), the radiation arriving at the observation point can be split in two parts: one is proportional to the product of the power a test dipole emits into the far field and the temperature of the surroundings; the second part is proportional to the dipole radiation power absorbed by the object and the metal temperature. At the sub-wavelength distances of interest for this paper, one can show that the second part is dominant and that the error made in using the same temperature for metal and surroundings is small. The equilibrium calculation we have focused on here is then sufficient.

\section{Acknowledgements}

This work has been supported by the European Union (project ACQP, contract IST-2001-38863) and Universität Potsdam (graduate school 'Confined Reaction and Interactions in Soft Matter'). We thank the quantum optics group in Potsdam for creating a stimulating environment.

\section{APPENDIX A: UNIFORM APPROXIMATION}

The integral over $k$ in Eq. (14) can be performed analytically if the power-law approximations of Table $\square$ are used for the reflection coefficients. We split the integration range at the crossing points between the power laws and sum the contributions. The full expression is cumbersume, and we quote here only the most complicated case, the thin layer in the range $k_{0} \ll k \ll 1 / \delta$ (first column of Table I). The integral can be 
handled with the formula

$$
\begin{aligned}
& \int_{k_{1}}^{k_{2}} \mathrm{~d} k \frac{(-1+(1+\mathrm{i}) k \delta) k \mathrm{e}^{-2 k d}}{1+\mathrm{i} k t} \\
& =(1-\mathrm{i}) \frac{\delta}{4 t d^{2}} \Gamma\left(2,2 k_{1} d, 2 k_{2} d\right) \\
& \quad+\frac{f}{2 d} \Gamma\left(1,2 k_{1} d, 2 k_{2} d\right) \\
& \quad+\frac{\mathrm{i} f}{t} \mathrm{e}^{-2 \mathrm{i} d / t} \Gamma\left(0,2 k_{1} d-2 \mathrm{i} d / t, 2 k_{2} d-2 \mathrm{i} d / t\right)
\end{aligned}
$$

where $t=\delta^{2} / h$ and $f=(1+\mathrm{i}) \delta^{2} / t+\mathrm{i} / t$. In this case, $k_{1}=k_{0}$ and $k_{2}=(t \delta)^{-1 / 2}$. The incomplete gamma function is defined by

$$
\begin{aligned}
\Gamma(n, b, a) & =\Gamma(n, b)-\Gamma(n, a) \\
\Gamma(n, a) & =\int_{a}^{\infty} \mathrm{d} t t^{n-1} \mathrm{e}^{-t}
\end{aligned}
$$

Using the asymptotics of the gamma function ${ }^{37}$, we get the power laws in Table II The logarithmic behaviour arises from

$$
a \ll 1: \quad \Gamma(0, a) \approx-\log a .
$$

\section{APPENDIX B: INCOHERENT SUMMATION}

We outline here the adaptation of the incoherent summation method of Ref 23 to two dimensions. The thermal spectrum of the current density is given at low frequencies $\left(\hbar \omega \ll k_{B} T\right)$ by

$$
\left\langle j^{*}(\mathbf{x} ; \omega) j\left(\mathbf{x}^{\prime} ; \omega^{\prime}\right)\right\rangle=2 \pi \delta\left(\omega-\omega^{\prime}\right) 2 k_{B} T \sigma(\mathbf{x} ; \omega) \delta\left(\mathbf{x}-\mathbf{x}^{\prime}\right)
$$

This spectrum is already integrated over a unit length in the $z$-direction (parallel to the current) along which the current density is assumed to be uniform (two-dimensional geometry). In this formulation, $\sigma$ is (the real part of) the $3 \mathrm{D}$ conductivity that we assume local, as reflected by the spatial $\delta$ correlation. We only take into account currents parallel to the $z$-direction. Each current element generates a magnetic field in the $x y$-plane that we compute in the magnetostatic approximation and ignoring the presence of the embedding metal. The latter point is the key approximation made. This gives a magnetic noise spectrum (integrated over a unit length along $z$ ) of the order of

$$
S_{B}=\frac{\mu_{0} k_{B} T}{\omega \delta^{2}}
$$

with cross correlations given by

$$
\begin{array}{r}
\mathcal{B}_{i j}(\mathbf{x} ; \omega)=\frac{S_{B}}{\pi^{2}}\left(\delta_{i j}(\operatorname{tr} Y)-Y_{i j}\right) \\
Y_{i j}(\mathbf{x})=\int_{V} \mathrm{~d} x_{1}^{\prime} \mathrm{d} x_{2}^{\prime} \frac{\left(x_{i}-x_{i}^{\prime}\right)\left(x_{j}-x_{j}^{\prime}\right)}{\left|\mathbf{x}-\mathbf{x}^{\prime}\right|^{4}}
\end{array}
$$

where $V$ is the volume occupied by the metal. The 'geometrical tensor' $Y_{i j}$ is dimensionless (a specific 2D property) and depends only on the ratio of observation distance and object size. It does not involve the skin depth, of course.

For a microstructure with rectangular cross section, an observer located above the center of the structure sees a noise power

$$
\begin{aligned}
\frac{\mathcal{B}_{x x}(d ; \omega)}{S_{B}}= & \frac{1}{2 \pi^{2}}\left[\left[\arctan \left(\frac{x^{\prime}}{y-y^{\prime}}\right)\right]_{x^{\prime}=-\frac{w}{2}}^{\frac{w}{2}}\right]_{y^{\prime}=-h}^{0} \\
& +\frac{1}{2 \pi^{2}}\left[\left[\operatorname{Im} \operatorname{Li}_{2}\left(\frac{\mathrm{i} x^{\prime}}{y-y^{\prime}}\right)\right]_{x^{\prime}=-\frac{w}{2}}^{\frac{w}{2}}\right]_{y^{\prime}=-h}^{0}(\mathrm{~B} 5) \\
\frac{\mathcal{B}_{y y}(d ; \omega)}{S_{B}=} & \frac{1}{2 \pi^{2}}\left[\left[\arctan \left(\frac{y-y^{\prime}}{x^{\prime}}\right)\right]_{x^{\prime}=-\frac{w}{2}}^{\frac{w}{2}}\right]_{y^{\prime}=-h}^{0} \\
& +\frac{1}{2 \pi^{2}}\left[\left[\operatorname{Im} \operatorname{Li}_{2}\left(\frac{\mathrm{i} x^{\prime}}{y-y^{\prime}}\right)\right]_{x^{\prime}=-\frac{w}{2}}^{\frac{w}{2}}\right]_{y^{\prime}=-h}^{0}
\end{aligned}
$$

where $\operatorname{Li}_{n}(\cdot)$ is the polylogarithm and we have used the notation

$$
\left[[f(u, v)]_{u=a}^{b}\right]_{v=c}^{d} \equiv f(a, c)-f(a, d)-f(b, c)+f(b, d)
$$

1 J. Nenonen, J. Montonen, and T. Katila, Rev. Sci. Instr. 67, 2397 (1996).

2 J. A. Sidles, J. L. Garbini, W. M. Dougherty, and S.-H. Chao, Proc. IEEE 91, 799 (2003).

${ }^{3}$ R. Folman, P. Krüger, J. Schmiedmayer, J. H. Denschlag, and C. Henkel, Adv. At. Mol. Opt. Phys. 48, 263 (2002).

4 J. Fortágh and C. Zimmermann, Rev. Mod. Phys. 79, 235 (2007).

${ }^{5}$ E. M. Purcell, Phys. Rev. 69, 681 (1946).

${ }^{6}$ G. S. Agarwal, Phys. Rev. A 11, 230 (1975).

7 T. Varpula and T. Poutanen, J. Appl. Phys. 55, 4015 (1984).

${ }^{8}$ R. R. Chance, A. Prock, and R. Silbey, in Advances in Chemical Physics XXXVII, edited by I. Prigogine and S. A. Rice (Wiley \&
Sons, New York, 1978), pp. 1-65.

9 W. L. Barnes, J. mod. Optics 45, 661 (1998).

10 S. Scheel, P.-K. Rekdal, P. L. Knight, and E. A. Hinds, Phys. Rev. A 72, 042901 (2005).

11 C. Henkel, Eur. Phys. J. D 35, 59 (2005), topical issue on "Atom chips: manipulating atoms and molecules with microfabricated structures".

12 L. Rogobete and C. Henkel, Phys. Rev. A 70, 063815 (2004).

${ }^{13}$ M. Nieto-Vesperinas, Scattering and Diffraction in Physical Optics (Wiley \& Sons, New York, 1991).

${ }^{14}$ R. F. Harrington, Field computation by moment methods (IEEE Press, Piscataway, 1993). 
15 C. Rockstuhl, M. G. Salt, and H. P. Herzig, J. Opt. Soc. Am. A 20, 1969 (2003).

${ }^{16}$ L. D. Landau, E. M. Lifshitz, and L. P. Pitaevskii, Electrodynamics of continuous media, 2nd ed. (Pergamon, Oxford, 1984).

17 S. M. Rytov, Y. A. Kravtsov, and V. I. Tatarskii, Elements of Random Fields, Vol. 3 of Principles of Statistical Radiophysics (Springer, Berlin, 1989).

18 D. Polder and M. V. Hove, Phys. Rev. B 4, 3303 (1971).

19 B. Zhang et al., Eur. Phys. J. D 35, 97 (2005), topical issue on "Atom chips: manipulating atoms and molecules with microfabricated structures".

${ }^{20}$ C. Henkel, S. Pötting, and M. Wilkens, Appl. Phys. B 69, 379 (1999), selected papers of the Heidelberg 1999 DPG meeting.

${ }^{21}$ S.-A. Biehs, D. Reddig, and M. Holthaus, Eur. Phys. J. B 55, 237 (2007).

${ }^{22}$ S. Bauer, Am. J. Phys. 60, 257 (1992).

${ }^{23}$ C. Henkel and S. Pötting, Appl. Phys. B 72, 73 (2001), selected papers of the Bonn 2000 DPG meeting.

24 E. Tuncer and D. P. Neikirk, Electr. Lett. 29, 2127 (1993).

25 J. Reichel, Appl. Phys. B 74, 469 (2002).

26 Y.-J. Lin, I. Teper, C. Chin, and V. Vuletić, Phys. Rev. Lett. 92, 050404 (2004).
27 V. Dikovsky, Y. Japha, C. Henkel, and R. Folman, Eur. Phys. J. D 35, 87 (2005), topical issue on "Atom chips: manipulating atoms and molecules with microfabricated structures".

${ }^{28}$ D. M. Harber, J. M. McGuirk, J. M. Obrecht, and E. A. Cornell, J. Low Temp. Phys. 133, 229 (2003).

29 B.-S. K. Skagerstam, U. Hohenester, A. Eiguren, and P. K. Rekdal, Phys. Rev. Lett. 97, 070401 (2006).

${ }^{30}$ M. C. Leung, J. Stat. Phys. 2, 305 (1970).

31 J. Tignon, T. Hasche, D. S. Chemla, H. C. Schneider, F. Jahnke, and S. W. Koch, Phys. Rev. Lett. 84, 3382 (2000).

32 K. L. Kliewer and R. Fuchs, Adv. Chem. Phys. 27, 355 (1974).

33 P. J. Feibelman, Progr. Surf. Sci. 12, 287 (1982).

${ }^{34}$ G. W. Ford and W. H. Weber, Phys. Rep. 113, 195 (1984).

35 C. Henkel, K. Joulain, R. Carminati, and J.-J. Greffet, Opt. Commun. 186, 57 (2000).

${ }^{36}$ M. Antezza, L. P. Pitaevskii, and S. Stringari, Phys. Rev. Lett. 95, 113202 (2005).

37 Handbook of Mathematical Functions, ninth ed., edited by M. Abramowitz and I. A. Stegun (Dover Publications, Inc., New York, 1972). 